\title{
A Modification of Polin's Variety *
}

\author{
Keith Kearnes $\quad$ Matthew Valeriote ${ }^{\dagger}$
}

January, 1999

\begin{abstract}
In this note we settle a question posed by Hobby and McKenzie in [2] on the nature of locally finite equational classes which satisfy some nontrivial congruence identity.
\end{abstract}

We settle problem \#14 from [2] by exhibiting a locally finite equational class $\mathcal{V}$ which omits types $\mathbf{1}$ and $\mathbf{5}$ and which satisfies some non-trivial congruence identity, but which contains a finite algebra having a type 4 minimal set with a nonempty tail. For background to this problem, the reader is encouraged to consult Chapter 9 of [2] and the paper [1] by Day and Freese on Polin's Variety.

Loosely stated, Polin's variety consists of algebras created by replacing the points of an (external) boolean algebra $\mathbf{B}$ by a family of (internal) boolean algebras $\left\{\mathbf{B}_{a}: a \in \mathbf{B}\right\}$ so that whenever $a \geq b$ in $\mathbf{B}$, there is a homomorphism $\xi_{b}^{a}$ from $\mathbf{B}_{a}$ to $\mathbf{B}_{b}$. These homomorphisms are compatible in the sense that if $a \geq b \geq c$ then $\xi_{c}^{a}=\xi_{b}^{a} \circ \xi_{c}^{b}$. The algebras come equipped with operations which allow one to recover both the internal and external boolean algebras.

Our example is a simple modification of this idea wherein we replace the internal boolean structures by distributive lattices having a distinguished largest element 1 . The proof that the resulting equational class satisfies some nontrivial congruence identity is practically the same as that presented by Day and Freese for Polin's variety in [1]. The tame congruence theoretic properties of the class referred to earlier can easily be established.

*1991 Mathematical Subject Classification. Primary 08B05; Secondary 08A30.

${ }^{\dagger}$ Support of NSERC is gratefully acknowledged 
Let $\mathbf{B}$ be a boolean algebra, $\mathbf{S}$ a function which assigns for each $a \in$ $B$ a distributive lattice $\mathbf{S}(a)$ with a largest element 1 , and $\left\langle\xi_{b}^{a}: \mathbf{S}(a) \rightarrow\right.$ $\mathbf{S}(b) \mid a \geq b$ in $\mathbf{B}\rangle$ a system of lattice homomorphisms such that $\xi_{a}^{a}=i d_{\mathbf{S}(a)}$ and if $a \geq b \geq c$ then $\xi_{c}^{a}=\xi_{c}^{b} \circ \xi_{b}^{a}$.

Define $\mathbf{A}(\mathbf{S}, \mathbf{B})$ to be the algebra with universe $\cup_{b \in B}\{b\} \times S(b)$ and having the following fundamental operations:

- $(a, s) \wedge(b, t)=\left(a \wedge b, \xi_{a \wedge b}^{a}(s) \wedge \xi_{a \wedge b}^{b}(t)\right)$,

- $(a, s) \vee(b, t)=\left(a \wedge b, \xi_{a \wedge b}^{a}(s) \vee \xi_{a \wedge b}^{b}(t)\right)$,

- $(a, s)^{\prime}=\left(a^{\prime}, 1\right)$.

Call such an algebra a boolean-by-distributive algebra, and let $\mathcal{B} / \mathcal{D}$ be the closure under isomorphism of the class of all such algebras. It is not difficult to see that this class is closed under subalgebras and direct products. Setting $\mathcal{V}$ to be the equational class generated by $\mathcal{B} / \mathcal{D}$ we have:

Theorem $1 \mathcal{V}$ is a locally finite equational class which satisfies the following congruence identity:

$$
x \wedge(y \vee z) \leq y \vee(x \wedge(z \vee(x \wedge y)))
$$

Proof. The corresponding results for Polin's variety are established in sections 2 and 7 of [1]. The proofs presented there can be used, almost without change, to prove our theorem.

Theorem $\mathbf{2} \mathcal{V}$ omits types $\mathbf{1}$ and $\mathbf{5}$ and contains a finite algebra $\mathbf{A}$ which has a type $\mathbf{4}$ minimal set with nonempty tail.

Proof. Since $\mathcal{V}$ satisfies a nontrivial congruence identity then by Theorem 9.18 of [2], it omits types $\mathbf{1}$ and $\mathbf{5}$. As an exercise, the reader can check that $\mathcal{V}$ actually omits type $\mathbf{2}$ as well.

Let $\mathbf{A}$ be the boolean-by-distributive algebra $\mathbf{A}(\mathbf{S}, \mathbf{B})$ where $\mathbf{B}$ is the two element boolean algebra, $\mathbf{S}(0)$ is a one element distributive lattice and $\mathbf{S}(1)$ is a two element distributive lattice. It can be easily checked that $\mathbf{A}$ has a unique nontrivial congruence $\alpha$, that the type of $\langle 0, \alpha\rangle$ is $\mathbf{4}$ and that $A$ is $\langle 0, \alpha\rangle$-minimal. This implies that the tail of $A$, considered as a $\langle 0, \alpha\rangle$-minimal set, is nonempty.

Further generalizations of Polin's construction have been investigated in the manuscript [3]. 


\section{References}

[1] A. Day and R. Freese. A characterization of identities implying congruence modularity, I. Canadian Journal of Mathematics, 32:1140-1167, 1980.

[2] D. Hobby and R. McKenzie. The Structure of Finite Algebras, volume 76 of Contemporary Mathematics. American Mathematical Society, 1988.

[3] N. Newrly and M. Valeriote. Some generalizations of Polin's variety. preprint, 1998.

Department of Mathematics

UNIVERSITY OF LOUISVILLE

LOUisville, KY 40292, USA.

Department of Mathematics and Statistics

MCMASTER UNIVERSITY

Hamilton, Ontario, Canada, L8S 4K1. 